\title{
Vortex-Resistance Hypothesis: Large Eddy Simulation of Turbulent Flow in Isolated Pool- Riffle Units
}

\author{
Hamed Dashtpeyma ${ }^{1, *}$, and Bruce MacVicar ${ }^{2}$ \\ ${ }^{1}$ Department of Civil and Environmental Engineering, University of Waterloo, Waterloo, Ontario, \\ Canada, N2L 3G1, Phone: 226-606-5422 \\ ${ }^{2}$ Department of Civil and Environmental Engineering, University of Waterloo, Waterloo, Ontario, \\ Canada, N2L 3G1
}

\begin{abstract}
By numerical simulations of turbulent flow in isolated poolriffle units with various riffle heights, four different types of vortices were found and named as follows: surface rollers (SR), corner rollers (CR), ramp rollers (CR), and axial tails (AT). Surface rollers are shaped on the flow surface due to submerged hydraulic jump or any obstacles in the forced poolriffle units. Corner rollers are shaped close to the corners near the walls at the pool head. Ramp rollers are formed at the bed of the channel on the ramp into the head of the pool. All kinds of vortices stretch in the streamwise direction as they travel to the downstream, which they are called axial tails. The simulations showed that all four types of vortices interact with each other, combine, amplify or cancel out each other as they travel downstream. The strength of vortices and how they interact result into different types of flow patterns. The surface rollers combine with corner rollers to make a jet like plunging flow near the pool bed. In other cases with lower riffle heights, ramp rollers tend to push the flow up, which in turn leads to higher turbulence near the bed and higher velocity near the flow surface (skimming flow). Moreover, if both surface rollers and ramp rollers have the similar strength (e.g., vorticity) and scale, the streamwise velocity profile has a peak around the middle of the flow, and minimum velocities near the bed and free surface. This flow pattern was named as "rifting flow." Based on these findings, a new hypothesis is proposed called 'vortex-resistance,' which states that the turbulent structures, by increasing the eddy viscosity and changing the pressure domain, act as an obstacle that steers the flow. Plunging and skimming flow can thus be understood as the products of different types of turbulent structures. These findings provide new clarifications to long-standing questions related to the hydraulics of pools and riffles.
\end{abstract}

\footnotetext{
*Corresponding author: hdashtpe@,uwaterloo.ca
} 


\section{Introduction}

Pools and riffles are common morphologic units in rivers and restoration projects. The deeper parts of undulations in the bed are called pools, whereas the shallower parts are riffles. They can be shaped naturally or artificially to control the stream energy, improve fish habitats, or control the sediment transport rate. Previous studies in the field, experimental flumes, and numerical studies have highlighted the complex interaction between the morphology, turbulence, and flow pattern. One of the most questionable facts in pool-riffle hydrodynamics is their formation and maintenance mechanism, which is possibly the result of higher bed shear stress in the pools. One of the most famous hypotheses in pool-riffle hydrodynamics is "velocity reversal" [1]. It says that with increasing flow discharge, the near-bed velocity in riffles increases at a lower rate than the near-bed velocity in pools such that there is a point that the near-bed velocity in the pool and riffle are equal, with higher shear stress projected for in pools at higher discharges [2]. This latter condition has not been consistently observed in various experimental analysis or the field, perhaps due to the difficulty of measuring at high water levels. This hypothesis can help to explain the formation of pools and riffles for few types of rivers because only a few studies in the field have found a reversal flow $[3,4]$. Still, other researchers showed that the likelihood and the magnitude of the reversal velocity were functions of width variations between the riffle and pool [5]. More recently, researchers have tried to restructure the reversal flow hypothesis by introducing the concept of lateral flow convergence [6,7]. However, most of the researchers in this field tried to resolve this confusion by time-averaged results without considering the effects of turbulence; therefore, they do not consider the dynamic effects of flow structures. A series of lab experiments [8] $[9,10]$ took a different approach by considering the pool tail as convective accelerating flow (CAF) and the pool head as convective decelerating flow (CDF), which zones as perturbations for turbulence generation. They showed that in some cases, velocity could be higher near the free-surface and turbulence higher near the bed, meaning that higher bed shear stress could be the result of higher turbulence instead of higher near-bed mean velocity. Despite the progress in the field and lab experiments, the characteristics and significance of turbulent structures that are generated over the riffle and in the pool remain poorly understood. In this research, we use large eddy simulations (LES) of turbulent flow in an isolated pool-riffle with different riffle heights to illustrate how vortices are created, dissipated, and interact with each other to form the overall patterns of flow and turbulence that have been observed.

\section{Methodology}

\subsection{Model Geometry}

The model geometry is based on the geometry of flume experiment case R40P240 completed at the University of Illinois, Urbana-Champaign [11]. This model is a simplified geometry of one pool and riffle unit. The simulated flume in the numerical model is $6 \mathrm{~m}$ long with riffle and pool lengths of $0.4 \mathrm{~m}$ and $2.4 \mathrm{~m}$, respectively (Fig. 1). For the simulated runs presented here, riffle height was varied as shown in Table 1 . The Froude number in the pool was 0.2 for all cases. Froude numbers in the riffle were calculated at the top downstream edge from simulated models, and they were the same as analytical calculation. Flow depth at the middle of the pool and inlet was $0.12 \mathrm{~m}$, and the inlet bulk velocity was $0.21 \mathrm{~m} / \mathrm{s}$. X, Y, and Z are streamwise, vertical and spanwise coordinates, respectively. 


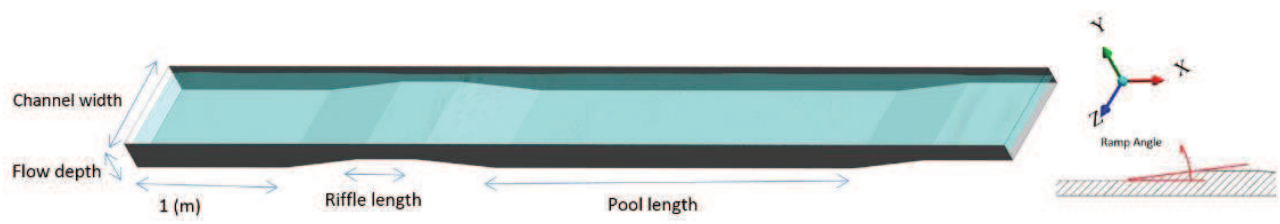

Fig. 1. Model Geometry

Table 1. Information of cases

\begin{tabular}{ccccccc}
\hline $\begin{array}{c}\text { Case } \\
\text { name }\end{array}$ & $\begin{array}{c}\text { Riffle } \\
\text { Height }\end{array}$ & $\begin{array}{c}\text { Ramp } \\
\text { Angle }\end{array}$ & $\begin{array}{c}\text { Inlet } \\
\text { velocity } \\
\left(\mathrm{U}_{\mathrm{o}}\right)\end{array}$ & $\begin{array}{c}\text { Flow } \\
\text { depth } \\
\text { (h) }\end{array}$ & $\begin{array}{c}\text { Fr at } \\
\text { riffle }\end{array}$ & $\begin{array}{c}\text { Fr at } \\
\text { pool }\end{array}$ \\
\hline $\mathrm{R} 45$ & $0.045 \mathrm{~m}$ & $4.95^{\circ}$ & $0.21 \mathrm{~m} / \mathrm{s}$ & $0.12 \mathrm{~m}$ & 0.5 & 0.2 \\
$\mathrm{R} 50$ & $0.050 \mathrm{~m}$ & $5.49^{\circ}$ & $0.21 \mathrm{~m} / \mathrm{s}$ & $0.12 \mathrm{~m}$ & 0.6 & 0.2 \\
$\mathrm{R} 55$ & $0.055 \mathrm{~m}$ & $6.04^{\circ}$ & $0.21 \mathrm{~m} / \mathrm{s}$ & $0.12 \mathrm{~m}$ & 0.7 & 0.2 \\
$\mathrm{R} 60$ & $0.060 \mathrm{~m}$ & $6.58^{\circ}$ & $0.21 \mathrm{~m} / \mathrm{s}$ & $0.12 \mathrm{~m}$ & 1.0 & 0.2 \\
$\mathrm{R} 65$ & $0.065 \mathrm{~m}$ & $7.13^{\circ}$ & $0.21 \mathrm{~m} / \mathrm{s}$ & $0.12 \mathrm{~m}$ & 1.1 & 0.2 \\
\hline
\end{tabular}

\subsection{Numerical Method}

The software that was used is ANSYS CFX Version 16.2. The mesh generating tool is ICEM Version 16.2 and post-processing was done with CFD-POST Version 16.2 and MATLAB R2017b. Large eddy simulation (LES) was used to capture information on turbulence generation, evolution and dissipation in pools and riffles. Because of smooth and non-slip walls and bed, LES Smagorinsky was chosen turbulence closure scheme. Previous studies have shown that LES is one of the best methods for modelling open channel, transient, and turbulent flow [12]. LES is an eddy-resolving technique that is suitable for such cases where hydrodynamic behaviour is controlled by large flow structures (structures with high kinetic energy). More information about the concept and formulation of LES can be found in reference [12]. The model is 3D with structured mesh. The total time for transient modelling is $250 \mathrm{~s}$, and all of the results between $50 \mathrm{~s}$, and $250 \mathrm{~s}$ were recorded. The first $20 \mathrm{~s}$ were not reliable because of initial condition effects. All of the suggestions in reference [12] were considered for meshing. The machine has dual CPUs; each has 10 cores of $2.3 \mathrm{GHz}$ with CPU model of Intel(R) Xeon(R) 2650 V3 and 192 GB RAM. A total number of elements was $32.5 \times 10^{6}$, and minimum size of them in the near-wall region was $1 \times 10^{-3} \mathrm{~m}$.

\section{Validation}

The time average of transient numerical results (case R50) was compared with the experimental data. Six profiles were chosen at the centreline at the middle of the riffle and pool; beginning of the ramp and the pool, and the quarter of the pool length. Streamwise time-average velocity $\left(\mathrm{U}_{\mathrm{x}}\right)$ showed consistency with experimental data in overall, especially 
in the favourable parts such as the end of the ramp and the middle of the pool for defining the flow pattern. As it is illustrated in the profiles, the numerical results provide more information about the flow near the bed (Fig. 2). Because of two reasons, the velocity profiles in the pool have lower velocity in the free surface region. First, there is a friction between air and water because of differences in their velocities. Second, the turbulence in the pool head causes fluctuations in flow elevation, during the time averaging process, the velocity of those points that were out of the water was considered as zero; therefore, the velocity near the surface has a lower average.
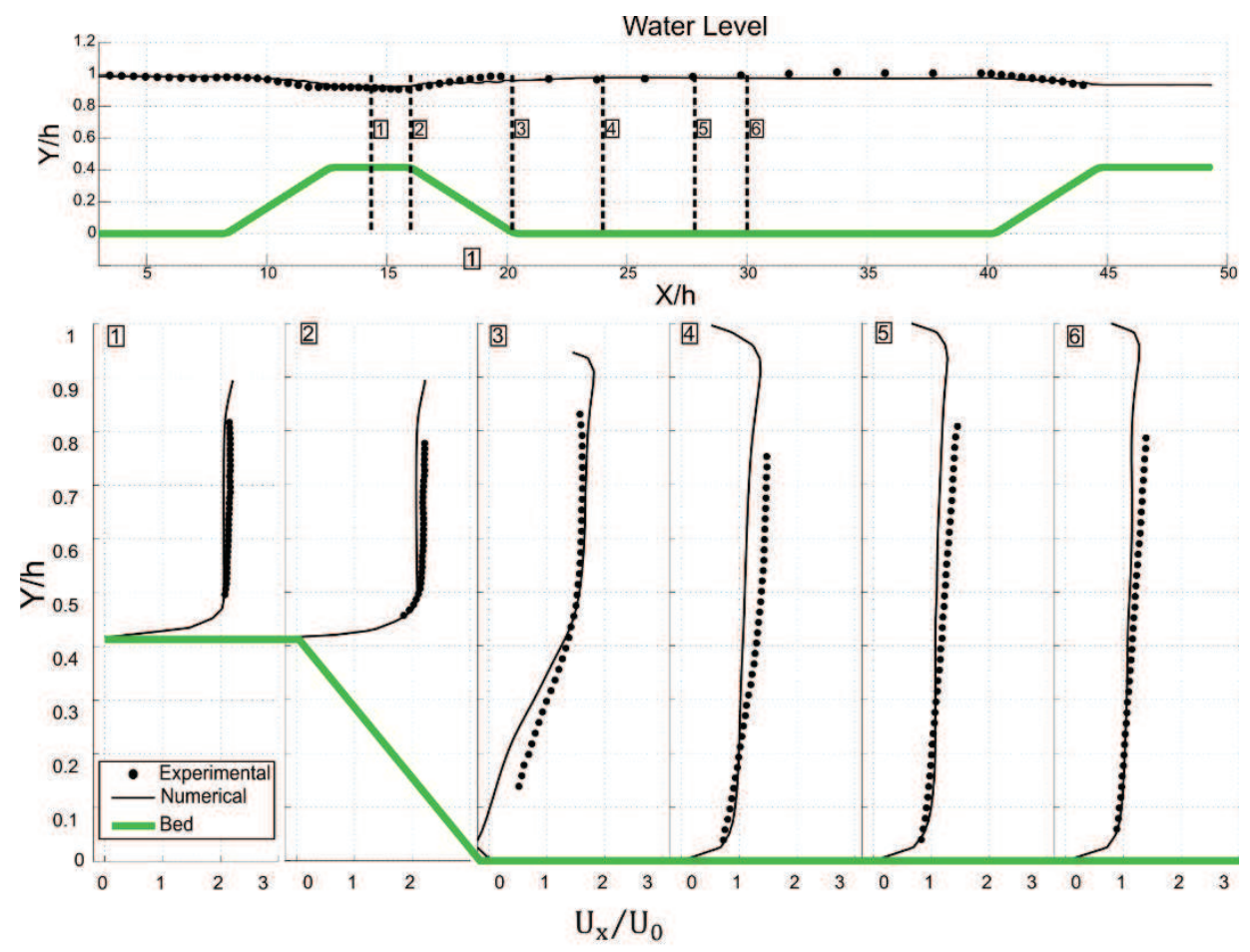

Fig. 2. Comparison of numerical results with experimental data

\section{Results}

\subsection{Detecting flow structures}

The Q-criterion was used to detect turbulent flow structures [13]. Instantaneous iso-surfaces of Q for the R45 (low riffle) and the R65 (high riffle) cases are shown in Figure 3. From this figure, it is possible to describe a number of different vortical structures in the flow, all of which originated in the decelerating flow between the riffle and the pool (CDF). Three types of vortices were detected in CDF zone, in all cases. There were sets of vortices in the corner near the wall at the pool head, which we called them corner rollers (CR). They were breaking into smaller vortices as they were travelling to the downstream. By checking the vorticity in those regions, it was figured out that $\mathrm{CR}$ vortices do not have a fixed spinning direction. Corner rollers were larger and stronger in cases with higher riffle height. In R45 a set of vortices near the bed in the ramp at the pool head was observed. We called these vortices as ramp rollers (RR). The negative vorticity in $\mathrm{Z}$ direction shows that these vortices are spinning counter-clockwise (in XY plane they rotate from left to right). They quickly break into 
smaller scale as they travel to the downstream. In R65 there were no ramp rollers, but there were vortices near the free surface at the pool head. These vortices had positive Z-vorticity which means they were spinning clockwise in opposite direction of ramp rollers. These rollers were shaping near the surface due to changing the state of flow from super-critical at the top of the riffle to sub-critical in the pool. Because of the Froude number between 1 and 1.3 , the hydraulic jump was shaped as surface undulation with surface rollers (SR). Surface rollers were combining with corner rollers and amplifying them. Case R65 had powerful SR, and after the combination of SR and CR, very strong vortices were shaped at the pool-head. All of the generated turbulent structures at the head of the pool start to energy cascading and breaking into smaller scales as they travel to the downstream in the form of long tubes, which are called axial tails (AT). When they become small enough, viscosity can dissipate their kinetic energy and change it into internal energy.

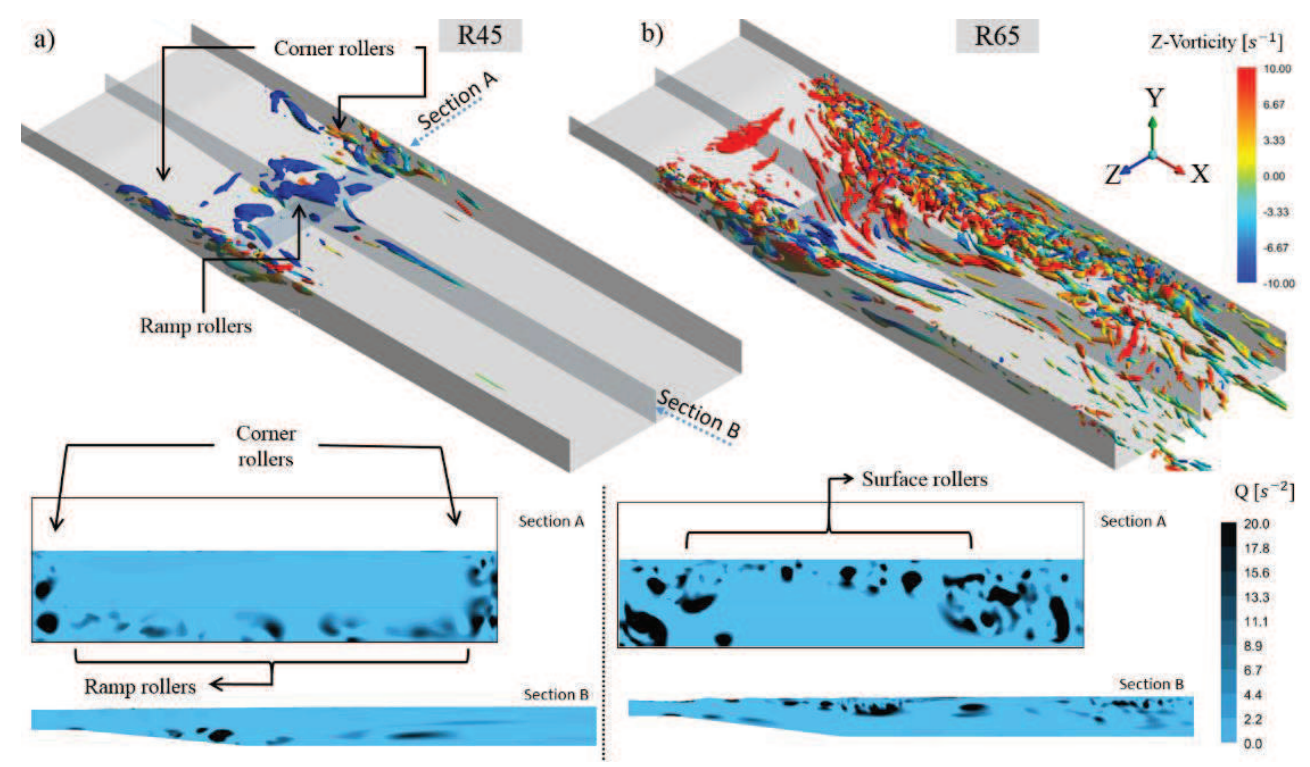

Fig. 3. Instantaneous flow structures in cases a) R45, and b) R65

\subsection{Vortex-resistance hypothesis}

For simplifying the turbulence modelling, Boussinesq (1877) [14] suggested eddy-viscosity method. He proposed that the effects of turbulent fluctuations on mean flow are the same as mixing effects of viscous forces; therefore, by adding a term, which is eddy-viscosity $\left(\mu_{t}\right)$, to dynamic viscosity $\left(\mu_{o}\right)$ the effects of turbulence is considered (equ 1).

$$
\mu=\mu_{o}+\mu_{t}
$$

The formulation of eddy-viscosity for LES Smagorinsky can be found in [12]. Eddy-viscosity is not a true viscosity like dynamic viscosity $\left(\mu_{o}\right)$, but an apparent viscosity due to turbulence. It is a turbulent form of resistance. They are similar because they represent a resistance to movement. By using the physical interpretation of eddy-viscosity method, it can be figured out that in those regions with higher eddy-viscosity, viscous forces can act as an obstacle resisting the mean flow and steering it. Because in open-channel flows turbulence is mostly generated by breakage and stretching of vortices, eddy-viscosity is mostly higher in those regions. Fig. 4a shows an example of the instantaneous ratio of eddy-viscosity over dynamic viscosity at the pool head for all cases. At the synced time Fig. $4 \mathrm{~b}$ shows the contour and 
vector of velocity and the core of vortices, which are shaded with black. As it is illustrated, eddy-viscosity is much higher in those regions where vortices are, and in some cases, it makes the flow up to ten times more viscous. Fig. 4b shows that in cases R60 and R65, those regions with higher viscosity near the free-surface (SR) resist the flow to travel freely near the freesurface, while those near the bed (RR) push the flow to skim the free-surface in cases R45 and R50. Based on these assumptions, and observations, the vortex-resistance hypothesis is suggested for a better understanding of vortices rule in the shaping of flow patterns.

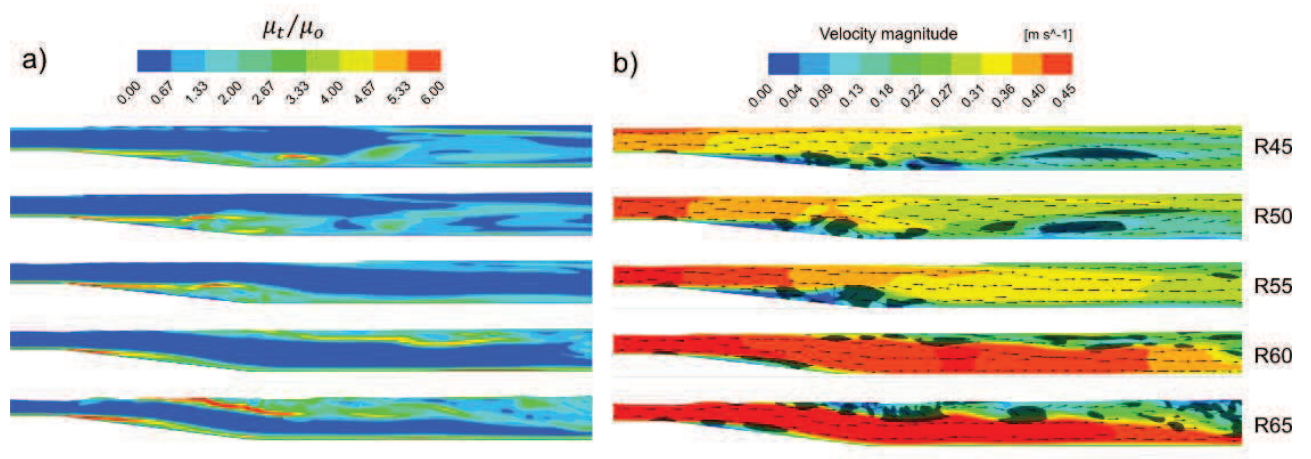

Fig. 4. a) Ratio of eddy-viscosity over dynamic viscosity contour at longitudinal cross-section, b) Contours and vectors of instantaneous velocity with shade of vortex core (Q)

\subsection{Effects of vortex-resistance on flow pattern}

By using the vortex-resistance hypothesis, three types of flow pattern were detected in poolriffle units with different riffle heights. The strength of vortices and how they interact result in different resistance to the mean flow which shapes the flow patterns differently. Fig. 5 shows the time-average velocity in different cases. Where flow is close to critical in riffles (Cases R60 and R65), the surface rollers and corner rollers restrict the flow and shape it as a jet-like plunging flow near the pool bed (Fig. 5 and Fig. 3b). In other cases (R45 and R50) with lower riffle heights, ramp rollers and corner rollers act as an obstacle near the bed and walls, which in turn leads to a higher velocity near the flow surface, which is called "skimming flow". Moreover, if both surface rollers and ramp rollers have the similar strength (e.g., vorticity) and scale, the streamwise velocity profile has a peak around the middle of the flow, and minimum velocities near the bed and free-surface (R55). This flow pattern was named as "rifting flow." 


\section{$U / U_{o}$}

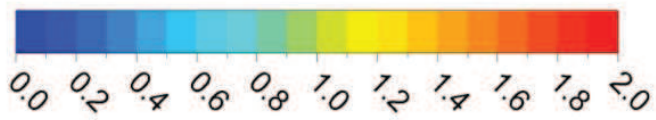

R45

R50

R55

$\mathrm{R} 60$

R65

Fig. 5. Contours of time average velocity for different cases

\section{Conclusion}

Based on large eddy simulation of flow pattern in isolated pool-riffle units with various riffle heights, a new hypothesis is proposed called 'vortex-resistance,' which states that the turbulent structures, by increasing the eddy viscosity and changing the pressure domain, act as an obstacle that steers the flow. If the vortices near the bed in the pool head push the flow up and make it skim near the surface, the flow pattern is called skimming flow. Moreover, if the vortices near the surface are shaped, they resist the flow and make the flow plunge into the pool. This type of flow was named as plunging flow. In balanced conditions, which vortices near the bed and the free-surface exert similar resistance to the flow, the flow will have the maximum velocity around the middle of channel depth. This flow pattern was called rifting flow. The vortex-resistance hypothesis may help to resolve the debate $([1,3,8,11,15-$ 17]) on the flow pattern in pool-riffle units. The results presented here show that a range of flow patterns are possible, and disagreements in the literature may be the result of observations from fundamentally different conditions.

\section{References}

1. E.A. Keller and J.L. Florsheim, Earth Surf. Process. Landforms, 18, 733-740 (1993).

2. E.A. Keller, Geol. Soc. Am. Bull., 82, 753 (1971).

3. D.M. Thompson, Pool-Riffle, in: Treatise Geomorphol., Elsevier, (2013), pp. 364 378.

4. G.L. Heritage and D.J. Milan, Catena, 58, 235-257 (2004).

5. S.N. Wilkinson, R.J. Keller, and I.D. Rutherfurd, Earth Surf. Process. ..., 29, 737753 (2004).

6. M.L. MacWilliams, J.M. Wheaton, G.B. Pasternack, R.L. Street, and P.K. Kitanidis, Water Resour. Res., 42, 1-21 (2006). 
7. A.M. Sawyer, G.B. Pasternack, H.J. Moir, and A.A. Fulton, Geomorphology, 114, 143-160 (2010).

8. B.J. MacVicar and C.D. Rennie, Water Resour. Res., 48, W02503 (2012).

9. B. MacVicar and J. Best, J. Geophys. Res. Earth Surf., 118, 1850-1863 (2013).

10. B.J. MacVicar, L. Obach, and J.L. Best, Large-Scale Coherent Flow Structures in Alluvial Pools, in: Coherent Flow Struct. Earth's Surf., John Wiley \& Sons, Ltd, Chichester, UK (2013), pp. 243-259.

11. B. MacVicar and L. Obach, J. Hydraul. Eng., 141, 4015025 (2015).

12. C.J. Keylock, G. Constantinescu, and R.J. Hardy, Geomorphology, 179, 1-20 (2012).

13. Y. Dubief and F. Delcayre, J. Turbul., 1, 1-22 (2000).

14. J. Boussinesq, Mem. Présentés Par Divers Savants Acad. Sci. Inst. Fr, 23, 5-6 (1877).

15. B.J. MacVicar and A.G. Roy, Water Resour. Res., 43, W12401 (2007).

16. B.J.J. MacVicar and A.G. Roy, Geomorphology, 125, 445-456 (2011).

17. D.M. Thompson, E.E. Wohl, and R.D. Jarrett, Phys. Geogr., 17, 142-156 (1996). 